\title{
Shouting strengthens voluntary force during sustained maximal effort through enhancement of motor system state via motor commands
}

Yudai Takarada ( $\sim$ takarada@waseda.jp )

Waseda University

Daichi Nozaki

University of Tokyo

\section{Research Article}

Keywords:

Posted Date: March 8th, 2022

DOI: https://doi.org/10.21203/rs.3.rs-1344023/v2

License: (c) (i) This work is licensed under a Creative Commons Attribution 4.0 International License.

Read Full License 


\section{Abstract}

Previous research indicates that shouting during momentary maximal exertion effort potentiates the maximal voluntary force through the potentiation of motor cortical excitability. However, the muscular force-enhancing effects of shouting on sustained maximal force production remain unclear. We investigated the effect of shouting on the motor system state by examining motor evoked potentials in response to transcranial magnetic stimulation applied over the hand area of the contralateral primary motor cortex (M1) during sustained maximal voluntary contraction, and by assessing handgrip maximal voluntary force. We observed that shouting significantly increased handgrip maximal voluntary force and reduced the silent period. Our results indicate that shouting increased handgrip voluntary force during sustained maximal exertion effort through the reduced silent period. This is the first objective evidence that the muscular force-potentiating effect of shouting during maximal force exertion is associated with the potentiation of motor system activity produced by the additional drive of shouting operating on the motor system (i.e., shouting-induced excitatory input to M1).

\section{Introduction}

It has been established that force levels of maximal voluntary contraction (MVC), in which a person believes their effort to be maximal, fluctuate constantly. Ikai and Steinhaus (1) proposed that the MVC is limited by psychological inhibiting mechanisms. The maximal voluntary force has been found to be increased by various manipulations such as the sound of a gunshot (1), hypnotic suggestion (1), shouting (1, 2), verbal encouragement (3), and motivational goal-priming (4).

Recently, we found that shouting increased the handgrip maximal voluntary force during brief nonfatiguing maximal effort through the reduced motor cortical inhibition, associated with potentiation of pupil-linked neuromodulatory system activity (5). Motor cortical inhibition in fatigued muscle has been also reported during sustained or repeated maximal effort, and the strength of this inhibition is substantially larger than that in unfatigued muscle during brief non-fatiguing maximal effort (6). If shouting has a motor cortical inhibition-reducing effect, as found during a brief MVC in brief nonfatiguing maximal effort (5), it would be expected to reduce motor cortical inhibition during sustained MVC in fatiguing maximal effort. This hypothesis is favored by previous evidence that shouting increases the maximal voluntary force of fatigued muscle during repeated maximal effort once a minute for $30 \mathrm{~min}$ (1).

Of note, in the shouting protocols used to increase MVC in several previous researches $(1,2,5)$, shouting occurred immediately prior to or almost simultaneously with muscular force exertion. Thus, shoutinginduced excitatory input to the motor system did not occur after muscular force exertion, making it impossible to examine the effect of shouting-induced motor commands on the motor system state as distinct from the effects of force exertion-induced motor commands. We further hypothesized that shouting following force exertion in the above sustained maximal effort would be expected to enhance 
the maximal voluntary force through potentiation of the motor system. Because we consider that producing a shout in itself is requisite for the muscular force-enhancing effect of shouting.

Here, we investigated the influence of shouting on the motor system state by examining motor evoked potentials (MEPs) in response to transcranial magnetic stimulation (TMS) applied over the hand area of the contralateral primary motor cortex (M1) during sustained MVC with intermittent shouting following force exertion. We also investigated the pupil-linked neuromodulatory system state by examining pupil size $(7,8)$, and by assessing handgrip maximal voluntary force. Our results indicated that shouting can increase the force level of MVC through the reduction of motor cortical inhibition during sustained MVC. This is the first objective evidence provides evidence that the muscular force-enhancing effect of shouting in maximal force exertion is associated with the enhancement of motor system activity, produced by the additional drive of shouting operating on the motor system (M1).

\section{Methods}

\section{Power Analysis}

We conducted an a priori power analysis to determine the required sample size for this experiment. We designed this experiment to have $80 \%$ power for detecting the effect sizes we previously found for the influence of motivational goal-priming on the motor system and action (0.46-0.64, Cohen's $d)(4,5,7)$ and/or pupil diameter $(0.50-0.61$, Cohen's $d)(7,8)$, using a significance level of $5 \%$. We used G*Power $3.1 \circledR$ (Institut für Experimentelle Psychologie, Düsseldorf, Germany) to compute the required sample size of the current study, which was 11 participants.

\section{Participants and Procedures}

Sixteen healthy Japanese right-handed individuals, as evaluated using the Edinburgh Handedness Inventory (9), participated in the study. The participants were 16 men, with a mean age \pm standard deviation of $20.1 \pm 1.6$ years. All participants provided both written and verbal informed consent. The study was conducted in accordance with the Declaration of Helsinki. All participants were university students who reported no strength training history, indicating that they had not received training regarding the exertion of the maximal force generated briefly by a muscle or group of muscles at a specified speed. Participants were randomly assigned to one of two groups ( $n=8$ for each group). Each group underwent two sessions of tests consecutively with at least a 3-min interval between sessions (see Participants and Procedures for details). The experimental procedures complied with relevant laws and institutional guidelines, and were approved by the Human Research Ethics Committee of the Faculty of Sport Sciences of Waseda University (approval number: 2020-411).

Experiments were designed to examine the influence of a self-generated shout on handgrip maximal voluntary force, pupillary size, and MEPs in the flexor carpi ulnaris (FCU) muscle in response to TMS (see TMS for details). Each experiment consisted of two sessions (shouting only and MVC), which were spaced at least 3 min apart on the same day. The MVC session consisted of three conditions (pre-MVC, 
sustained MVC, and post-MVC), each with a duration of approximately $120 \mathrm{~s}$, which performed in that order (Fig. 1). In the shouting-only session, participants were asked to shout five times with a 30-s intershouting interval, as loudly as possible for 1-2 s when a cue was given by an experimenter ("One, two, three, start") in a quiet voice. In the pre-MVC condition of the MVC session, participants performed five brief MVCs (1-2 s duration) when an experimenter gave a cue ("One, two, three, squeeze") with a $30 \mathrm{~s}$ inter-squeeze interval. After a rest of approximately $3 \mathrm{~min}$, participants performed a 2-min sustained MVC with five periods of intermittent shouting (sustained MVC condition) according to experimental instructions on a screen in front of them (see Pupil Diameter Measurement for details). A previous study reported that 2-min sustained MVC induces a progressive, exercise-induced decline in voluntary activation of a muscle (central fatigue) (6). Participants were not given advance notice of when the sustained MVC condition would terminate. However, they were informed in advance that they would be asked to shout several times during the sustained MVC condition, as in the shouting-only session. Immediately following the sustained MVC, a series of brief contractions was performed, which was the same process as that in the pre-MVC condition (post-MVC condition). The total experimental period lasted approximately $20 \mathrm{~min}$. Visual feedback and verbal encouragement were given throughout all handgrip contractions. In all conditions, participants were asked to keep their heads still and to keep their hands on their lap in a sitting posture while maintaining as much stability in the core as possible, and to keep viewing the screen in front of them.

\section{Pupil Diameter Measurement}

Pupil diameter was measured using a TalkEye Lite system (Takei Scientific Instruments Co., Ltd., Tokyo, Japan). An image around the pupil was obtained using a camera employing near-infrared light-emitting diodes and a video graphics array $(640 \times 480)$ (built-in digital signal processor) camera module (NCM03$\mathrm{V}$, Nippon Chemi-Con Corporation, Tokyo, Japan). Banalization processing was performed on each image, and the pupil diameter was then measured according to the methods described by Wang et al. (10). Changes in pupil size were estimated by the area of the pupil $(7,8)$ while participants viewed the screen in front of them in all conditions. We calculated the average pupil area (dots) for $500 \mathrm{~ms}$ before each TMS (see TMS) during squeezing of a handgrip device (see Handgrip Force Measurement) in the pre-MVC, post-MVC, and sustained MVC conditions of the MVC session.

The following steps were taken to exclude the impact of experimenter expectations for participant responses and measurements as much as possible, and to objectively estimate the effect of shouting in the sustained MVC condition. 1) The experimental procedure of the sustained MVC condition was conducted automatically using a $60 \mathrm{~Hz}$ cathode ray tube screen to display the text, and the experimental procedure was created using software designed for psychological experiments (Inquisit 3 Desktop Edition, Millisecond Software, Seattle, WA, USA). 2) All participants were instructed to follow the starting and stopping signals on the screen. 3) Pupil diameter measurements were automatically performed using a specially designed device with an eye-capturing camera to obtain an image around the pupil. Consequently, the paradigm used in the present study was less susceptible to experimenter bias 
compared with outcome measurements that have typically been used for examining the maximal voluntary force in previous studies (11).

All word stimuli were displayed in black $\left(20.5 \mathrm{~cd} / \mathrm{m}^{2}\right.$ : mean value of five measurements of luminance with an LS160 luminance meter; Konica Minolta, Inc., Tokyo, Japan) on a white screen $\left(123.2 \mathrm{~cd} / \mathrm{m}^{2}\right)$ during the experimental procedure. Immediately before the word presentation, the color of the screen was momentarily white without any black words. The pupil diameter may have transiently decreased because of the increase in luminance caused by the white screen with a maximum luminance of $128.5 \mathrm{~cd} / \mathrm{m}^{2}$. Thus, we were unable to completely eliminate the possibility that this transient change in luminance affected pupil diameter. However, any effect on the results would be likely to be minimal, because this phenomenon was present for all participants and conditions.

\section{Handgrip Force Measurement}

Force was measured using a handgrip device (KFG-5-120-C1-16, Kyowa Electronic Instruments, Tokyo, Japan). In the baseline and recovery MVC conditions, participants performed five brief MVCs (1-2 s duration) on a cue given by an experimenter ("One, two, three, squeeze") with a 30-s inter-squeeze interval. In the sustained MVC condition, the experimental instructions on the screen asked participants to squeeze the handgrip device with the right (dominant) hand with maximum effort when the word "squeeze" appeared on the display, and to stop squeezing when the word disappeared. The handgrip device was fixed to the right thigh with an elastic band so that the device did not move when it was squeezed by the participant. The maximal values of the exerted force were averaged from the $500-\mathrm{ms}$ steady state of the force curve before each TMS (Fig. 2A, B) across the five trials for the pre- and postMVC conditions, and of each trial for the sustained MVC condition (see TMS for details). These were defined as the handgrip maximal voluntary force.

\section{TMS}

In the shouting-only session, and the pre-MVC, sustained MVC, and post-MVC conditions of the MVC session, single-pulse TMS was administered via a stimulator (M2002, Magstim, Whitland, UK) using a double figure-eight-shaped coil (4150-00 Double $70 \mathrm{~mm}$ Alpha Coil, Magstim) with a maximum magnetic field strength of $1.55 \mathrm{~T}$. Each participant sat upright with their elbows bent in front of them, and their hands resting on their thighs. The TMS coil was then positioned over the finger area of the left M1, which was determined as the area with the lowest resting motor threshold (rMT). This was defined as the area for which MEPs with peak-to-peak amplitudes greater than $50 \mu \mathrm{V}$ were induced in the FCU muscle $(5,7,12,13)$ in at least five of 10 trials when participants were fully relaxed with their eyes closed (14). The coil position was stabilized throughout the experiment using a coil stand made from multiple products (Manfrotto Distribution KK, Tokyo, Japan). The optimal scalp position of M1 was marked directly onto the scalp with a black marker pen. The positioned coil was monitored continuously to maintain consistent positioning throughout the experiment. The rMTs ranged from $40-80 \%$ of the maximum stimulator output, and the stimulus intensity for each participant was set at almost the same intensity as 
their rMT while shouting in the shouting-only session. The stimulus intensity was set from $70-90 \%$ of the maximum stimulator output during handgrip force exertion. Stimulation was manually delivered over the target site during each shouting period (1-2 s duration) and each brief MVC (1-2 s duration), with a 30-s inter-squeeze interval (Fig. 2A, B). The timing of TMS was different for each episode of shouting in the shouting condition of the shouting-only session, and each brief MVC in the pre- and post-MVC conditions of the MVC session. Thus, MEPs were recorded five times for each condition (shouting or pre- or post$M V C)$. The stimulation was automatically delivered 10 times at $12 \mathrm{~s}$ intervals for the 2 min sustained MVC condition, during which shouting (sustained MVC condition with shouting) or no shouting (sustained MVC condition without shouting) were alternated every $12 \mathrm{~s}$. Thus, MEPs were recorded 10 times. Ten MEPs were classified into five sets (S1-S5) according to the timeline, each of which included two MEPs with or without shouting (Fig. 1). The order of the shouting in the sustained MVC condition was counterbalanced so that eight participants shouted in the odd-numbered TMS trials (Experimental group 1) and the other participants shouted in the even-numbered TMS trials (Experimental group 2). The TMS intensity was fixed for each participant. Surface electromyography (EMG) was obtained from the right FCU muscles via bipolar silver surface electrodes (band pass, $15 \mathrm{~Hz}-10 \mathrm{kHz}$ ) using the tendon-belly method $(5,7,13)$.

\section{Background EMG and MEP Measurement and Analysis}

We measured the peak-to-peak amplitude of each MEP (Fig. 3A, B). We calculated the averaged waveform of MEP (an average of five recordings) in the pre-MVC and post-MVC conditions (see TMS for details). To measure background EMG (bEMG), a rectified EMG signal of the period approximately 100 ms before TMS was integrated, during which the force was kept at the maximum force level (Fig. 2A, B).

The duration of the silent period was taken as the time interval from the stimulus artifact to the return of continuous EMG $(15,16)$ (Fig. 3B). When it was difficult to determine the end of the silent period (because voluntary EMG activity recovers gradually rather than abruptly), the end of the silent period was determined when the corresponding rectified EMG activity reached a value within two standard deviations of the rectified EMG signal of the period approximately $100 \mathrm{~ms}$ before TMS $(17,18)$.

\section{Sound Level}

Sound level in the shouting condition and the sustained MVC condition was measured using by a Digital Sound Level Meter (GM1356, Benetech, Guangdong, China), which was placed diagonally in front of the participant and to their left, at face level. The readings are in Decibel, A-weighted (dBA) units representing the sound level measured with the A-weighting network on the sound level meter. This instrument incorporates a type-1 microphone and records sound ranging from 30-130 dBA with a sensitivity index of $\pm 0.1 \mathrm{dBA}$ (sampling rate $20 \mathrm{~Hz}$ ). Fast impulse mode was used to record the readings.

\section{Statistical Analysis}

Statistically significant differences in MEP amplitude between resting state and the shouting condition, and sound level between the shouting and the sustained MVC conditions were investigated using paired 
$t$-tests. The maximal voluntary force, the duration of the silent period, MEP: bEMG ratios and pupil area in the sustained MVC condition were analyzed using repeated-measures three-way analyses of variance (ANOVAs) with experimental group (between-participants factor: (shouting in the odd-numbered TMS trials [later: group 1] or shouting in the even-numbered TMS trials [first: group 2]) (2)) $\times$ order of each set (within-participants factor: [shouting or no shouting] (2)) $\times$ set (within-participants factor: [1-5 sets] (5)). Greenhouse-Geisser corrections were applied when appropriate to adjust for non-sphericity, changing the degrees of freedom using a correction coefficient. A significance threshold of $p<0.05$ was chosen for all tests. A significance threshold of $p<0.05$ was selected for all tests.

\section{Results}

First, we ascertained that shouting enhanced the excitability of motor system activity: shouting (199.9 \pm $38.6 \mu \mathrm{V})$ significantly increased MEP amplitudes compared with those of $\mathrm{rMT}(77.7 \pm 10.8 \mu \mathrm{V})(t[15]=-$ $3.46, d=1.08 ; p=0.003$ ) (Fig. 3A). There were no significant differences in sound level between the shouting-only $(105.6 \pm 1.1 \mathrm{dBA})$ and the sustained MVC condition $(104.6 \pm 1.1)(t[15]=1.87, d=0.23 ; p=$ 0.08).

Several effects of shouting on behavior and motor system state were observed (Table 1), which were generally consistent with previously published findings (5). Shouting significantly increased handgrip maximal voluntary force, followed by a reduction of the silent period.

\section{Handgrip force}

Significant changes of the maximal voluntary force during the sustained MVC were observed when combined with shouting. Three-way ANOVA revealed that there was no significant second-order interaction $\left(F[4,56]=0.31 ; p=0.86\right.$; effect size: partial $\left.\eta^{2}=0.022\right)$ (Table 2). There were significant firstorder interactions between order (shouting or no shouting) and experimental group $(F[1,14]=13.2 ; p=$ 0.003; effect size: partial $\eta^{2}=0.48$ ), and between set $(1-5$ sets) and order (shouting or no shouting) $\left(F[4,56]=14.0 ; p=5.58 \times 10^{-8}\right.$; effect size: partial $\left.\eta^{2}=0.50\right)$. There were significant simple main effects of shouting on the maximal voluntary force in each experimental group (order of shouting [first]: $p=0.016$; order of shouting [later]: $p=0.031$ )(Fig. 4): shouting significantly increased the handgrip force level of sustained MVC.

\section{TMS}

Compared with no shouting, the duration of the silent period during handgrip MVC was reduced when combined with shouting. Three-way ANOVA revealed that there was no significant second-order interaction $\left(F[4,56]=0.47 ; p=0.75\right.$; effect size: partial $\left.\eta^{2}=0.033\right)$ (Table 3). There were significant firstorder interactions, between order (shouting or no shouting) and experimental group $(F[1,14]=17.0 ; p=$ 0.001 ; effect size: partial $\eta^{2}=0.55$ ). There were significant simple main effects of shouting on the maximal voluntary force in each experimental group (order of shouting [first]: $p=0.006$; order of shouting 
[later]: $p=0.020$ ) (Fig. 5). However, MEP amplitude revealed no significant changes between two orders (shouting vs. no shouting), as shown in Table 4. Concerning background (b)EMG, a three-way ANOVA revealed that there was no significant second-order interaction $(F[2.32,32.6]=1.61 ; p=0.21$; effect size: partial $\eta^{2}=0.10$ ) (Table 5). There were significant first-order interactions between order (shouting or no shouting) and experimental group $\left(F[1,14]=11.5 ; p=0.004\right.$; effect size: partial $\left.\eta^{2}=0.45\right)$, and between set $(1-5$ sets $)$ and experimental group $(F[4,56]=3.14 ; p=0.021$; effect size: 0.18$)$ (Fig. 5). Significant changes of bEMG during sustained MVC were observed when combined with shouting in the same manner as observed in the maximal voluntary force.

\section{Pupil area}

Three-way ANOVA revealed that there was no significant second-order interaction, and no significant firstorder interaction (Table 6). However, there was a significant main effect of order (shouting vs. no shouting) on pupil area $\left(F[1,14]=8.86 ; p=0.01\right.$; effect size: partial $\left.\eta^{2}=0.38\right)$. Post hoc analyses with Bonferroni corrections for multiple comparisons demonstrated that there was a significant change between two orders (shouting vs. no shouting) at time point $3(p=0.005)$.

\section{Discussion}

The results revealed that shouting significantly increased the handgrip force level of sustained MVC, caused by a reduction of the silent period. Such an enhancing effect of shouting on handgrip MVC is generally consistent with the results of previous studies $(1,2,5)$. Our findings indicate that the motor system was more excitable during sustained muscular contraction paired with shouting, leading to the generation of additional muscular force in maximal exertion effort. These results show that the muscular force-potentiating effect of shouting in maximal force exertion is relevant to the potentiation of motor system activity, via the additional drive of shouting operating on M1.

The main finding was the remarkable potentiation effect of shouting on the handgrip maximal voluntary force during a 2-min period of sustained MVC, with an average increase of approximately $30 \%$ in the handgrip force. This increase rate is greater than the effect of shouting on the maximal voluntary force (15\%) during a brief MVC reported in our previous study (5). One potential reason for this discrepancy is a difference in the experimental period of muscular force exertion between the present study ( $2 \mathrm{~min}$ ) and the previous study (within $5 \mathrm{~s}$ ). It is conceivable that maximal force exertion for 2 min induces both peripheral and central fatigue, resulting in a marked decline in force production during a 2-min sustained MVC. Indeed, handgrip maximal voluntary force declined to approximately $70 \%$ of pre-MVC. This could be caused by a progressive, exercise-induced decline in voluntary activation of a muscle during sustained maximal effort, which attributes to impairment at sites proximal to the neuromuscular junction (6). Thus, the differences in the potentiating effects on MVC mentioned above may be relevant to the experimental period and fatigue of human voluntary contractions.

The muscular force-enhancing effect of shouting was accompanied by a reduced silent period. Changes in silent periods of longer than $100 \mathrm{~ms}$ in the hand muscles of healthy participants (19) are considered to 
reflect cortical inhibition (15). The cortical silent period originates largely from the M1 (15), in which GABAergic circuits are believed to produce the cortical silent period (20-22). The cortical silent period is used as a measure for assessing motor excitability (23). Thus, shouting transiently may enhance the activity of motor cortical neurons. At the same time, we must keep in mind that shouting may enhance the activity of spinal motor neurons, because bEMG during handgrip MVC significantly increased when combined with shouting. However, we were convinced that such an enhancement of the spinal motor neuronal activity may by caused by the enhancing effect of shouting on motor cortical neurons. Because analysis of covariance for continuous measurements adjusted for bEMG revealed that there was no significant interaction, between set $(1-5$ sets $)$ and bEMG $\left(F[9,140]=1.25 ; p=0.26\right.$; effect size: partial $\eta^{2}$ $=0.075)$; however, there was a significant difference of $y$-intercept $(p<0.001)$ : there was a significant reduction of SP between with and without shouting, irrespective of changes in bEMG. This notion is supported by recent evidence that shouting increases handgrip MVC through the reduction of motor cortical inhibition with no significant changes in bEMG (5).

We found that shouting led to a reduced silent period, and increased handgrip maximal voluntary force. Increased maximal voluntary force may have been brought about by the reduced motor cortical inhibition. These enhancement effects of shouting on handgrip MVC and motor system activity were observed when participants shouted while exerting the handgrip muscular force during maximal effort: shouting never occurred immediately prior to or almost simultaneously with force exertion. Thus, the additional drive of shouting operating on the motor system led to a reduced silent period, and increased maximal voluntary force. This result supported the present finding that producing a shout without handgrip muscular contraction enhanced the motor system state, indicating that the motor command for shouting is transmitted to the motor cortex, and in the final cortical stage of the motor execution program the motor command of shouting is devoted to M1. Therefore, shouting-induced excitatory input to M1 contributes to the muscular force-enhancing effect of shouting during maximal force exertion.

In spite of the reduced silent period, we never observed any changes in MEP amplitude during MVC; there were no statistically significant differences in MEP amplitudes between with and without shouting during handgrip MVC (Fig. 5B). The reason for this failure to detect any changes is because most of the M1 neurons may have already been recruited (24), leaving fewer neurons available to respond to TMS. Thus, the level of M1 neuron recruitment reaching a plateau during MVC might have overshadowed any differences in MEP amplitudes during MVC between with and without shouting.

No consistent enhancing effects of shouting during handgrip MVC on pupil area were observed in the present study (see Pupil area in Results for details). A recent study reported that shouting significantly increased the handgrip force level of a brief MVC for 1-2 s, followed by an increase in pupil size (5). One reason for this discrepancy is a difference in an experimental protocol concerning MVC between the present study (continuous) and the previous study (momentary). It is conceivable that there is no room for pupil dilation when shouting is performed throughout the maximal force exertion for $2 \mathrm{~min}$. In the current study, pupil area markedly increased immediately after the appearance of the word "squeeze" on the display, and remained at a high level until the end of squeezing; there was no significant difference in 
pupil area between the pre-MVC and the sustained MVC without shouting (Fig. 6; Table 1: Set 1 no-shout

in order of shouting [first] $)(t[7]=0.96, d=0.34 ; p=0.36)$. Thus, the differences in the influence of shouting on pupil area may be related to experimental force exertion protocol in maximal effort. It should be noted, however, that the current results do not necessarily imply that shouting never affects the pupil-linked neuromodulatory system state. The level of pupillary dilation reaching a plateau during MVC (25) might have overshadowed any differences in the pupil-linked neuromodulatory system state between with and without shouting.

We found that shouting led to a reduced silent period during MVC, and increased handgrip maximal voluntary force. Increased handgrip maximal force may have been brought about by the reduced motor cortical inhibition during maximal effort, through the additional drive of shouting operating on the motor system. These results indicate that the muscular force-potentiating effect of shouting in maximal force exertion is relevant to the potentiation of motor system activity, caused by the transmitted motor command of shouting to M1. In turn, this suggests that maximum volition does not cause the motor system to generate maximum activity. This is an active characteristic of the neural systems in the human brain. However, we do not know to what exact extent that spinal motor neuronal excitability involves in neural activity of the force-enhancing effect of shouting, a puzzle that remains to be solved. From the perspective of accessing the latent ability for human force exertion, it may be useful for future studies to examine whether devoting an additional motor command, such as shouting, to M1 improves the characteristics of neural system activity.

\section{Declarations}

\section{Acknowledgments}

This work was supported by a Grant-in-Aid for Scientific Research (C) from the Ministry of Education, Culture, Sports, Science and Technology in Japan (Projects 21K11532).

Author Contributions: The complete list of author contributions to the paper according to the CRediT model is as follows: 1. Conceptualization Y.T.; 2. Data curation Y.T.; 3. Formal analysis Y.T. and D.N. (supporting); 4. Grant acquisition Y.T.; 5. Investigation Y.T.; 6. Methodology Y.T.; 7. Project administration Y.T.; 8. Resources Y.T.; 9. Software Y.T. and D.N.; 10. Supervision Y.T.; 11. Validation Y.T.; 12. Visualization Y.T.; 13. Writing original draft Y.T.; 14. Writing-review and editing Y.T. and D.N; 15. Statistical Analysis Y.T. and D.N.

Competing Interest Statement: The authors declare no competing interests.

\section{References}

1. Ikai, M. \& Steinhaus, A. H. Some factors modifying the expression of human strength. J. Appl. Physiol. 16, 157-163 (1961). 
2. Welch, A. S. \& Tschampl, M. Something to shout about: a simple, quick performance enhancement technique improved strength in both experts and novices. J. Appl. Sport Psychol. 24, 418-428 (2012).

3. McNair, P. J., Depledge, J., Brettkelly, M. \& Stanley, S. N. Verbal encouragement: effects on maximum effort voluntary muscle action. Br. J. Sports Med. 30, 243--245 (1996).

4. Takarada, Y. \& Nozaki, D. Maximal voluntary force strengthened by the enhancement of motor system state through barely visible priming words with reward. PLoS ONE 9, e109422 (2014).

5. Takarada, Y. \& Nozaki, D. Shouting strengthens maximal voluntary force and is associated with augmented pupillary dilation. Sci. Rep. 11, 18419 (2021).

6. Gandevia, S. C., Allen, G. M., Butler, J. E. \& Taylor, J. L. Supraspinal factors in human muscle fatigue: Evidence for suboptimal output from the motor cortex. J. Physiol. 490, 529-536 (1996).

7. Takarada, Y. \& Nozaki, D. Motivational goal-priming with or without awareness produces faster and stronger force exertion. Sci. Rep. 8, 10135 (2018).

8. Takarada, Y. \& Nozaki, D. Pupil dilations induced by barely conscious reward goal-priming. Neuropsychologia 103, 69-76 (2017).

9. Oldfield, R. C. The assessment and analysis of handedness: the Edinburgh inventory. Neuropsychologia 9, 97-113 (1971).

10. Wang, J., Zhang, G. \& Shi, J. Pupil and glint detection using wearable camera sensor and nearinfrared LED array. Sensors 15, 30126-30141 (2015).

11. Nuzzo, J. L., Taylor, J. L. \& Gandevia, S. C. CORP: Measurement of upper and lower limb muscle strength and voluntary activation. J. Appl. Physiol. 126, 513-543 (2019).

12. Takarada, Y., Mima, T., Abe, M., Nakatsuka, M. \& Taira, M. Inhibition of the primary motor cortex can alter one's "sense of effort": effects of low-frequency rTMS. Neurosci. Res. 89, 54-60 (2014).

13. Takarada, Y., Ohki, Y. \& Taira, M. Effect of transient vascular occlusion of the upper arm on motor evoked potentials during force exertion. Neurosci. Res. 76, 224-229 (2014).

14. Rossini, P. M., et al., Non-invasive electrical and magnetic stimulation of the brain, spinal cord and roots: basic principles and procedures for routine clinical application. Report of an IFCN committee. Electroencephalogr. Clin. Neurophysiol. 91, 79-92 (1994).

15. Inghilleri, M., Berardelli, A., Cruccu, G. \& Manfredi, M. Silent period evoked by transcranial stimulation of the human cortex and cervicomedullary junction. J. Physiol. 466, 521-534 (1993).

16. Damron, L. A., Dearth, D. J., Hoffman, R. L. \& Clark, B. C. Quantification of the corticospinal silent period evoked via transcranial magnetic stimulation. J. Neurosci. Methods 173, 121-128 (2008).

17. Duclay, J., Pasquet, B., Martin, A \& Duchateau, J. Specific modulation of spinal and cortical excitabilities during lengthening and shortening submaximal and maximal contractions in plantar flexor muscles. J. Appl. Physiol. 117, 1440-1450 (2014).

18. Groppa, S., et al., A practical guide to diagnostic transcranial magnetic stimulation: report of an IFCN committee. Clin. Neurophysiol. 123, 858-882 (2012). 
19. Cantello, R., Gianelli, M., Civardi, C. \& Mutani, R. Magnetic brain stimulation: the silent period after the motor evoked potential. Neurology 42, 1951-1959 (1992).

20. Connors, B. W., Malenka, R. C. \& Silva, L. R. Two inhibitory postsynaptic potentials, and GABAA and GABAB receptor-mediated responses in neocortex of rat and cat. J. Physiol. 406, 443-468 (1988).

21. Werhahn, K. J., Kunesch, E., Noachtar, S., Benecke, R. \& Classen, J. Differential effects on motorcortical inhibition induced by blockade of GABA uptake in humans. J. Physiol. 517(Pt 2), 591597 (1999).

22. Siebner, H. R., Dressnandt, J., Auer, C. \& Conrad, B. Continuous intrathecal baclofen infusions induced a marked increase of the transcranially evoked silent period in a patient with generalized dystonia. Muscle Nerve. 21, 1209-1212 (1998).

23. Ziemann, U. \& Hallett, M. Basic neurophysiological studies with TMS. In: George MS, Belmaker RH, editors. Transcranial magnetic stimulation in neuropsychiatry. Washington, DC: American Psychiatry Press; 2000. p. 45-98.

24. Matthews, P. B. C. The effect of firings on the excitability of a model motoneurones and its implications for cortical stimulation. J. Physiol. 518, 867-882 (1999).

25. Zénon, A., Sidibé, M. \& Olivier, E. Pupil size variations correlate with physical effort perception. Front. Behav. Neurosci. 8, 286 (2014).

\section{Tables}

\begin{tabular}{|c|c|c|c|c|c|c|}
\hline & & Maximal voluntary force $(\mathrm{N})$ & Silent period (ms) & MEP amplitude (mV) & $\mathrm{bEMG}(\mu \mathrm{V} \cdot \mathrm{s})$ & Pupil area (dot) \\
\hline \multirow{13}{*}{$\begin{array}{l}\text { Experimental } \\
\text { group (later) }\end{array}$} & Pre-MVC & $282.2 \pm 18.5$ & $157.9 \pm 10.5$ & $1.12 \pm 0.10$ & $13.5 \pm 1.41$ & $2807.0 \pm 159.2$ \\
\hline & \multicolumn{6}{|l|}{ Sustained MVCs } \\
\hline & Set1 no-shout & $261.9 \pm 23.3$ & $155.1 \pm 16.2$ & $1.80 \pm 0.19$ & $10.5 \pm 0.85$ & $2696.9 \pm 204.3$ \\
\hline & shout & $240.7 \pm 17.0$ & $152.5 \pm 19.9$ & $1.58 \pm 0.17$ & $10.9 \pm 1.18$ & $2526.2 \pm 141.0$ \\
\hline & Set 2 no-shout & $155.3 \pm 16.1$ & $209.2 \pm 15.6$ & $1.92 \pm 0.20$ & $9.33 \pm 2.09$ & $2502.3 \pm 199.1$ \\
\hline & shout & $162.6 \pm 17.0$ & $163.3 \pm 25.9$ & $1.68 \pm 0.30$ & $9.35 \pm 2.46$ & $2415.8 \pm 203.2$ \\
\hline & Set 3 no-shout & $128.1 \pm 14.0$ & $191.7 \pm 26.0$ & $1.70 \pm 0.27$ & $7.97 \pm 2.58$ & $2542.3 \pm 196.9$ \\
\hline & shout & $139.4 \pm 12.2$ & $181.0 \pm 32.7$ & $1.51 \pm 0.23$ & $9.34 \pm 2.60$ & $2227.2 \pm 145.6$ \\
\hline & Set 4 no-shout & $106.9 \pm 8.1$ & $265.9 \pm 71.6$ & $1.78 \pm 0.22$ & $9.51 \pm 2.93$ & $2561.4 \pm 166.1$ \\
\hline & shout & $123.0 \pm 12.3$ & $165.1 \pm 32.0$ & $1.59 \pm 0.30$ & $11.4 \pm 1.87$ & $2398.2 \pm 159.8$ \\
\hline & Set 5 no-shout & $93.9 \pm 7.4$ & $180.4 \pm 16.5$ & $1.76 \pm 0.27$ & $6.20 \pm 1.78$ & $2655.0 \pm 185.0$ \\
\hline & shout & $106.0 \pm 10.5$ & $165.3 \pm 21.3$ & $1.62 \pm 0.22$ & $7.83 \pm 1.64$ & $2148.2 \pm 199.3$ \\
\hline & Post-MVC & $200.7 \pm 19.6$ & $151.7 \pm 12.0$ & $1.13 \pm 0.09$ & $12.0 \pm 1.46$ & $2748.6 \pm 138.6$ \\
\hline \multirow{13}{*}{$\begin{array}{l}\text { Experimental } \\
\text { group (first) }\end{array}$} & Pre-MVC & $298.7 \pm 17.5$ & $178.9 \pm 18.5$ & $0.72 \pm 0.10$ & $9.73 \pm 2.07$ & $2401.0 \pm 171.4$ \\
\hline & \multicolumn{6}{|l|}{ Sustained MVCs } \\
\hline & Set1 no-shout & $217.5 \pm 23.9$ & $257.1 \pm 65.5$ & $1.05 \pm 0.13$ & $8.21 \pm 1.00$ & $2315.0 \pm 177.4$ \\
\hline & shout & $301.0 \pm 30.6$ & $165.0 \pm 20.6$ & $0.92 \pm 0.24$ & $10.54 \pm 1.76$ & $2521.8 \pm 173.1$ \\
\hline & Set 2 no-shout & $158.3 \pm 14.3$ & $222.7 \pm 40.0$ & $1.16 \pm 0.14$ & $5.39 \pm 1.47$ & $2349.9 \pm 171.4$ \\
\hline & shout & $207.4 \pm 27.5$ & $170.8 \pm 20.7$ & $1.13 \pm 0.12$ & $7.83 \pm 1.48$ & $2327.5 \pm 122.6$ \\
\hline & Set 3 no-shout & $120.3 \pm 11.9$ & $180.2 \pm 21.9$ & $0.97 \pm 0.20$ & $3.78 \pm 0.88$ & $2241.0 \pm 166.1$ \\
\hline & shout & $185.3 \pm 25.8$ & $163.7 \pm 17.8$ & $1.12 \pm 0.16$ & $7.71 \pm 2.35$ & $2487.9 \pm 152.1$ \\
\hline & Set 4 no-shout & $102.7 \pm 7.05$ & $176.2 \pm 26.1$ & $0.98 \pm 0.097$ & $2.61 \pm 0.50$ & $2178.8 \pm 151.3$ \\
\hline & shout & $159.5 \pm 19.8$ & $188.3 \pm 16.6$ & $0.93 \pm 0.082$ & $6.96 \pm 1.67$ & $2375.8 \pm 207.0$ \\
\hline & Set 5 no-shout & $87.6 \pm 5.7$ & $229.0 \pm 23.8$ & $1.02 \pm 0.080$ & $3.50 \pm 0.57$ & $2286.3 \pm 236.6$ \\
\hline & shout & $126.0 \pm 15.5$ & $163.7 \pm 20.3$ & $1.15 \pm 0.16$ & $4.77 \pm 1.44$ & $2360.2 \pm 218.2$ \\
\hline & Post-MVC & $199.3 \pm 15.5$ & $162.4 \pm 18.1$ & $0.91 \pm 0.10$ & $7.65 \pm 0.80$ & $2333.7 \pm 198.4$ \\
\hline
\end{tabular}

Table 2. Repeated-measures three-way analyses of variance (ANOVAs) results for Maximal voluntary force. 


\begin{tabular}{|l|l|l|l|l|l|l|}
\hline Source of Variation & Sum of squares & Degree of freedom & Mean square & F-value & p-value & partial $\eta^{2}$ \\
\hline Group & 10.43 & 1 & 10.43 & 0.022 & 0.88 & 0.002 \\
Error & 6612.8 & 14 & 472.3 & & & \\
\hline Set & 21444.9 & 4 & 5361.2 & 56.8 & $4.44 \times 10^{-19}$ & 0.80 \\
Set x Group & 463.1 & 4 & 115.7 & 1.22 & 0.30 & 0.081 \\
Error & 5278.6 & 56 & 94.2 & & & \\
\hline Condition & 15.2 & 1 & 15.2 & 0.056 & 0.81 & 0.004 \\
Order x Group & 3593.6 & 1 & 3593.6 & 13.2 & 0.003 & 0.48 \\
Error & 3800.7 & 14 & & & & \\
\hline Set x Order & 5166.4 & 4 & 271.4 & & & \\
Set x Order x Group & 115.4 & 4 & 28.8 & 0.31 & 0.86 & 0.022 \\
Error & & & & & & \\
\hline
\end{tabular}

Table 3. Repeated-measures three-way analyses of variance (ANOVAs) results for Silent period.

\begin{tabular}{|l|l|l|l|l|l|l|}
\hline Source of Variation & Sum of squares & Degree of freedom & Mean square & F-value & p-value & partial $\eta^{2}$ \\
\hline Group & 0.003 & 1 & 0.003 & 0.068 & 0.79 & 0.005 \\
Error & 0.61 & 14 & 0.044 & & & \\
\hline Set & 0.008 & 2.15 & 0.004 & 0.43 & 0.66 & 0.003 \\
Set x Group & 0.039 & 2.15 & 0.018 & 2.11 & 0.13 & 0.13 \\
Error & 0.25 & 30.1 & 0.009 & & & \\
\hline Order & 0.001 & 1 & 0.001 & 0.16 & 0.69 & 0.012 \\
Order x Group & 0.060 & 1 & 0.060 & 17.0 & 0.001 & 0.55 \\
Error & & & & & & \\
\hline Set x Order & 0.049 & 14 & 0.004 & & & \\
Set x Order x Group & 0.007 & 4 & 0.012 & 3.21 & 0.019 & 0.18 \\
Error & & 4 & 0.002 & 0.47 & 0.75 & 0.033 \\
& 0.20 & 56 & & & & \\
\hline
\end{tabular}

Table 4. Repeated-measures three-way analyses of variance (ANOVAs) results for MEP amplitude. 


\begin{tabular}{|c|c|c|c|c|c|c|}
\hline Source of Variation & Sum of squares & Degree of freedom & Mean square & F-value & p-value & partial $\eta^{2}$ \\
\hline Group & 75704.0 & 1 & 75704.0 & 2.26 & 0.15 & 0.13 \\
\hline Error & 467497.1 & 14 & 33392.6 & & & \\
\hline Set & 8263.4 & 4 & 2065.8 & 1.60 & 0.18 & 0.10 \\
\hline Set x Group & 1319.2 & 4 & 329.8 & 0.25 & 0.90 & 0.018 \\
\hline Error & 72320.8 & 56 & 1291.4 & & & \\
\hline Order & 2282.2 & 1 & 2282.2 & 3.06 & 0.102 & 0.17 \\
\hline Order x Group & 2491.5 & 1 & 2491.5 & 3.34 & 0.089 & 0.19 \\
\hline Error & 10435.4 & 14 & 745.3 & & & \\
\hline Set x Order & 1898.3 & 4 & 474.6 & $\begin{array}{l}0.80 \\
1.00\end{array}$ & $\begin{array}{l}0.52 \\
0.41\end{array}$ & $\begin{array}{l}0.054 \\
0.067\end{array}$ \\
\hline $\begin{array}{l}\text { Set x Order x Group } \\
\text { Error }\end{array}$ & 2389.2 & 4 & 597.3 & & & \\
\hline
\end{tabular}

Table 5. Repeated-measures three-way analyses of variance (ANOVAs) results for bEMG.

\begin{tabular}{|l|l|l|l|l|l|l|}
\hline Source of Variation & Sum of squares & Degree of freedom & Mean square & F-value & p-value & partial $\eta^{2}$ \\
\hline Group & 373.9 & 1 & 373.9 & 0.066 & 0.80 & 0.005 \\
Error & 79348.1 & 14 & 5667.7 & & & \\
\hline Set & 36956.8 & 4 & 9239.2 & 9.67 & $5 \times 10^{-5}$ & 0.40 \\
Error & 12011.8 & 4 & 3002.9 & 3.14 & 0.021 & 0.18 \\
\hline Order & 53504.1 & 56 & & & & \\
Order x Group & 13000.1 & 1 & 455.4 & & & 0.23 \\
Error & 15766.6 & 14 & 13000.1 & 11.5 & 0.004 & 0.45 \\
\hline Set x Order & 834.4 & 2.32 & & & & \\
Set x Order x Group & 3303.1 & 2.32 & 358.2 & 0.40 & 0.69 & 0.028 \\
Error & 28621.3 & 32.60 & 1418.2 & 1.61 & 0.21 & 0.10 \\
\hline
\end{tabular}

Table 6. Repeated-measures three-way analyses of variance (ANOVAs) results for pupil area. 


\begin{tabular}{|c|c|c|c|c|c|c|}
\hline Source of Variation & Sum of squares & Degree of freedom & Mean square & F-value & $p$-value & partial $\eta^{2}$ \\
\hline Group & 3209.2 & 1 & 3209.2 & 2.99 & 0.10 & 0.17 \\
\hline Error & 14987.7 & 14 & 1070.5 & & & \\
\hline Set & 870.0 & 2.50 & 347.3 & 1.60 & 0.21 & 0.10 \\
\hline Set $x$ Group & 224.8 & 2.50 & 89.7 & 0.41 & 0.70 & 0.029 \\
\hline Error & & 35.0 & 216.8 & & & \\
\hline Order & 2427.9 & 1 & 2427.9 & 8.86 & 0.01 & 0.38 \\
\hline Order x Group & 67.2 & 1 & 67.2 & 0.24 & 0.62 & 0.17 \\
\hline Error & & 14 & & & & \\
\hline Set $\mathrm{x}$ Order & 403.6 & 2.26 & 177.9 & 0.84 & 0.45 & 0.057 \\
\hline Set x Order x Group & 433.6 & 2.26 & 191.2 & 0.90 & 0.42 & 0.061 \\
\hline Error & 6678.5 & 31.7 & 210.2 & & & \\
\hline
\end{tabular}

\section{Figures}

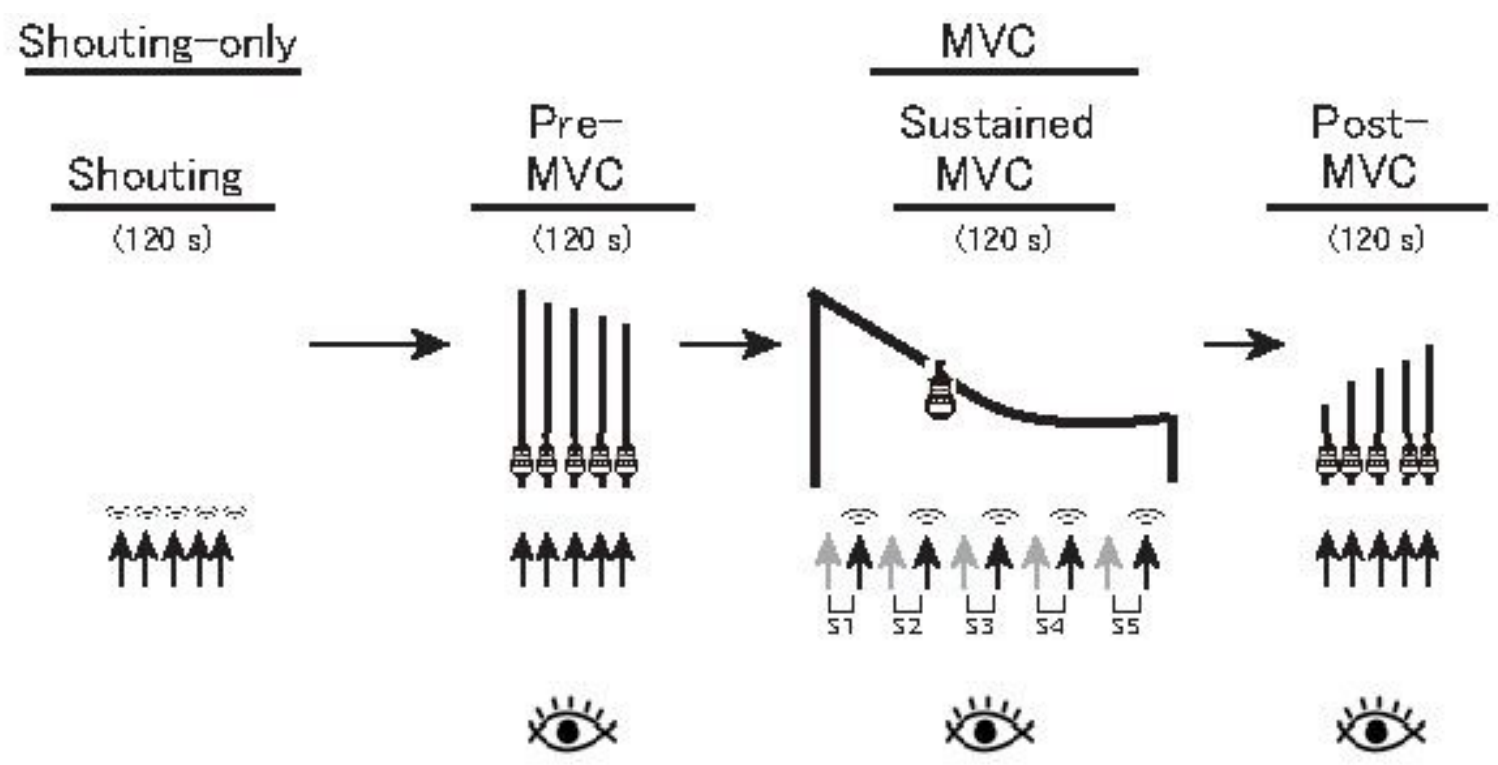

Sound level

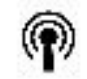

(

Figure 1

Experimental procedure. Each experiment consisted of two sessions (only

shouting and MVC). The MVC session consisted of three orders (pre-MVC, sustained MVC, and post$M V C)$, each with a duration of approximately $120 \mathrm{~s}$, which were performed in that order with a break of at least $3 \mathrm{~min}$ between orders. The total experimental time was approximately $20 \mathrm{~min}$. The timing of transcranial magnetic stimulation (TMS) is indicated by the arrow. Ten TMS during the sustained MVC 
session were classified into the five sets (S1 S5) according to the timeline. The order of shouting in the sustained MVC condition was counterbalanced so that eight participants shouted in the odd-numbered TMS trials (Experimental group 1) and the other participants shouted in the even-numbered TMS trials (Experimental group 2).

\section{Figure 2}

Typical recordings of handgrip force, and background electromyography (bEMG) of the flexor carpi ulnaris during the maximal voluntary contraction (MVC) of handgrip in pre- MVC condition in a single participant. The timing of transcranial magnetic stimulation is indicated by the arrow. The handgrip force declined when transcranial magnetic stimulation was delivered during the contraction, the timing of which was different in each contraction. (A) Handgrip force. (B) bEMG during handgrip contraction. 


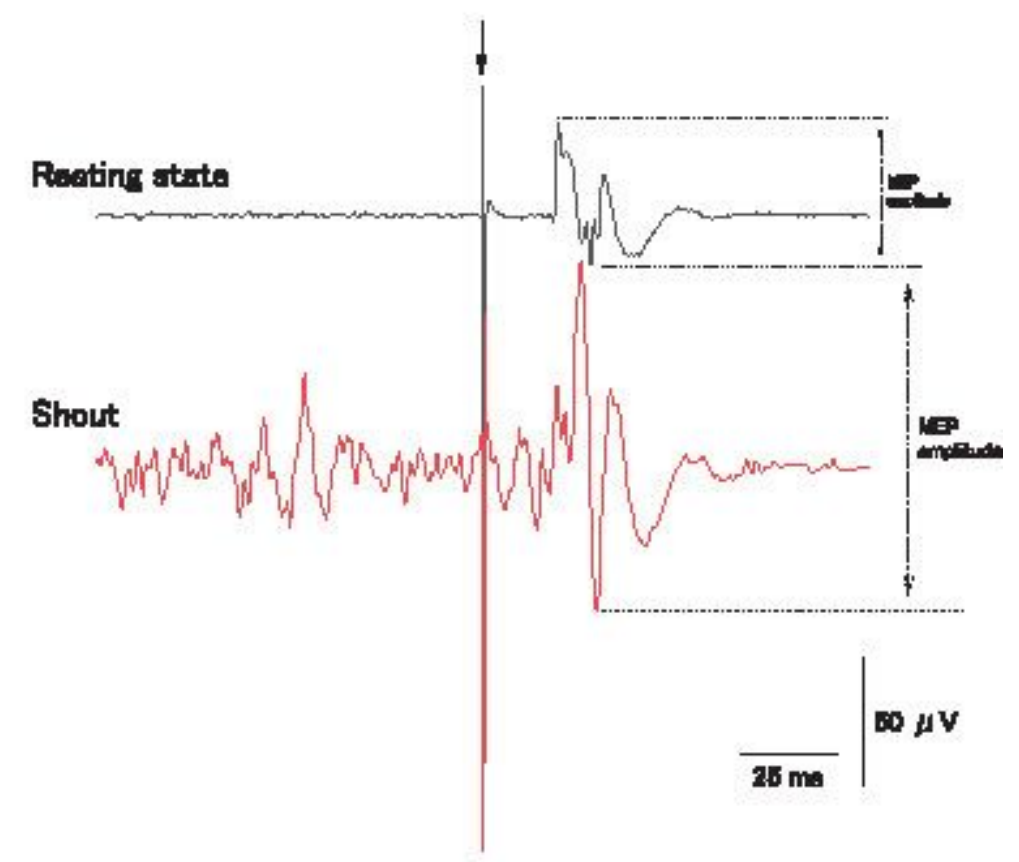

B

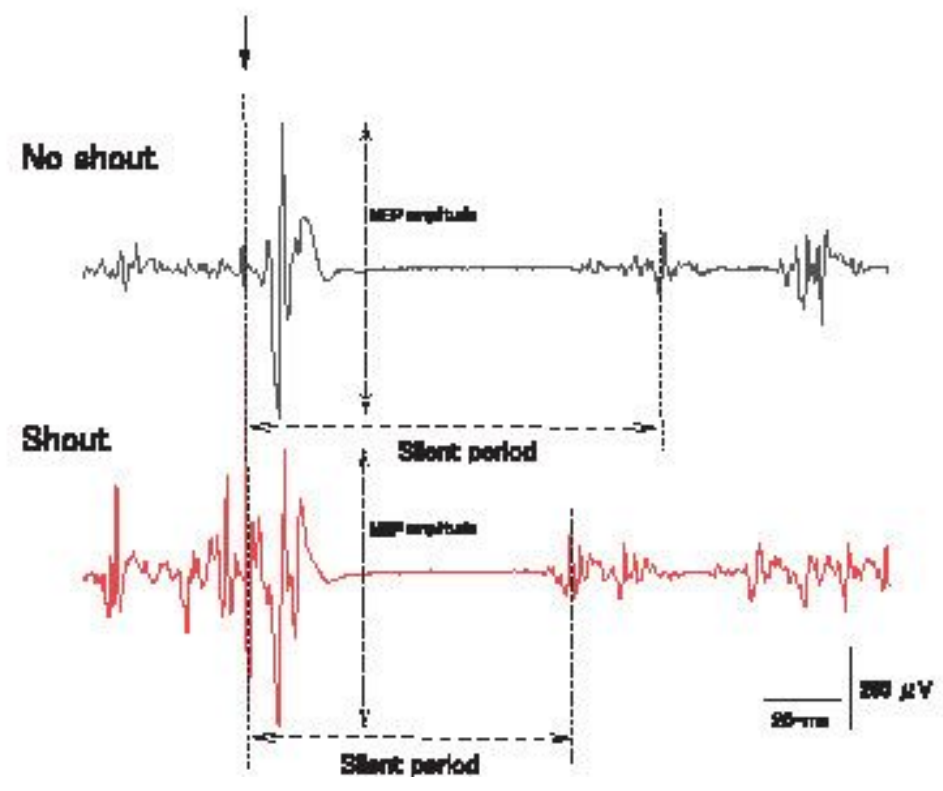

Figure 3

Typical motor evoked potential (MEP) waveforms of the flexor carpi ulnaris (FCU) during the maximal voluntary contraction force (MVC) of handgrip in a single participant. The timing of transcranial magnetic stimulation is indicated by the arrow. MEPs measured from the relaxed FCU muscle during the resting state and during shouting for the shouting-only condition in a single participant (A) and during handgrip contraction with or without shouting for the sustained MVC condition in a single participant (B). The bidirectional arrows indicate amplitudes of MEPs and duration of the silent period. 


\section{Figure 4}

Effects of shouting on the maximal voluntary force of handgrip. The maximal voluntary force for each condition (pre-MVCs, sustained MVC, and post-MVCs conditions) during the maximal voluntary contraction (MVC) of handgrip in each experimental group. Shouting significantly increased the maximal voluntary force of handgrip during a 2-min sustained MVC. Red cross indicates handgrip contraction with shouting. *Statistically significant difference between the handgrip contractions performed with and without shouting in shaded area. Data are expressed as the mean \pm standard error of the mean. 

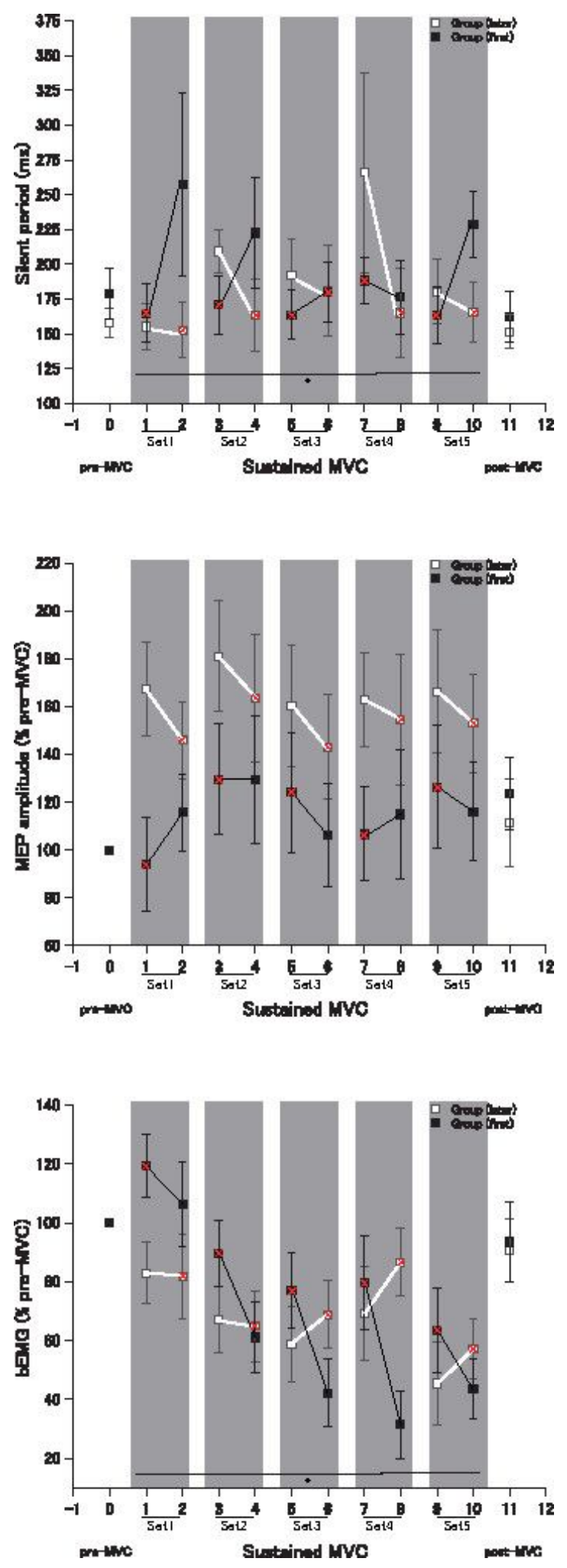

Figure 5

Effects of shouting on the silent period, motor evoked potential (MEP) amplitude and background (b) EMG. (A) Durations of the silent period for each condition (pre-MVC, sustained MVC, and post-MVC conditions) during the maximal voluntary contraction (MVC) of handgrip. (B) Amplitudes of MEPs of the flexor carpi ulnaris (FCU) for the three conditions (pre-MVC, sustained MVC, and post-MVC conditions) during the handgrip MVC. (C) Background (b) EMG activity of the FCU for the three conditions (pre-MVC, 
sustained MVC, and post-MVC conditions) during the handgrip MVC. Shouting significantly shortened the duration of the silent period, and significantly increased

bEMG during a 2 min sustained MVC. Red cross indicates handgrip contraction with shouting. $*$ Statistically significant difference between the contractions performed with and without shouting in shaded area. Data are expressed as the mean \pm standard error of the mean.

\section{Figure 6}

Effects of shouting on pupil area. Averaged pupil area (dots) for $500 \mathrm{~ms}$ before each transcranial magnetic stimulation during squeezing a handgrip device in the pre-MVC, post-MVC, and sustained MVC conditions in each experimental group. Red cross indicates handgrip contraction with shouting. *Statistically significant difference between the handgrip contractions performed with and without shouting in shaded area. Data are expressed as the mean \pm standard error of the mean. 